\title{
Preparation, Synergism, and Biocompatibility of in situ Liquid Crystals Loaded with Sinomenine and 5-Fluorouracil for Treatment of Liver Cancer
}

\author{
Jiaojiao $\mathrm{Cao}^{1, *}$ \\ Jie Huang ${ }^{l} * *$ \\ Shuangying Gui ${ }^{1,2}$ \\ Xiaoqin Chu ${ }^{1,2}$
}

'Department of Pharmaceutics, Anhui University of Chinese Medicine, Hefei, 2300 I2, People's Republic of China; ${ }^{2}$ Institute of Pharmaceutics, Anhui Academy of Chinese Medicine, Hefei, 230012, People's Republic of China

*These authors contributed equally to this work
Correspondence: Xiaoqin Chu

Master Instructor

Tel +86 I385692992।

Fax +86 55I-68129028

Email chuxq420@I63.com
Purpose: Transarterial chemoembolization is the preferred treatment for patients with middle and advanced-stage hepatocellular carcinoma (HCC); however, most hepatic artery embolization agents have various disadvantages. The purpose of this study was to evaluate phytantriol-based liquid crystal injections for potential use in treatment of HCC.

Methods: Using sinomenine (SN) and 5-fluorouracil (5-FU) as model drugs, three precursor in situ liquid crystal injections based on phytantriol (P1, P2, and P3) were prepared, and their in vitro biocompatibility, anticancer activity, and drug release investigated, to evaluate their feasibility for use in treatment of HCC. The properties of the precursor injections and subsequent cubic liquid crystal gels were observed by visual and polarizing microscopy, in an in vitro gelation experiment. Biocompatibility was evaluated by in vitro hemolysis, histocompatibility, and cytotoxicity assays.

Results: Precursor injections were colorless liquids that formed transparent cubic liquid crystal gels on addition of excess water. The three precursor injections all caused slight hemolysis, without agglutination, and were mildly cytotoxic. Histocompatibility experiments showed that P1 had good histocompatibility, while P2 and P3 resulted in strong inflammatory responses, which subsequently resolved spontaneously. In vitro anti-cancer testing showed that $\mathrm{SN}$ and 5-FU inhibited HepG2 cells in a time- and concentration-dependent manner and had synergistic effects. Further, in vitro release assays indicated that all three preparations had sustained release effects, with cumulative release of $>80 \%$ within $48 \mathrm{~h}$.

Conclusion: These results indicate that $\mathrm{SN}$ and 5-FU have synergistic inhibitory effects on HepG2 cells, which has not previously been reported. Moreover, we describe a biocompatible precursor injection, useful as a drug carrier for the treatment of liver cancer, which can achieve targeting, sustained release, synergistic chemotherapy, and embolization. These data indicate that precursor injections containing SN and 5-FU have great potential for use in therapy for liver cancer.

Keywords: carcinoma, hepatocellular, drug carriers, delayed-action preparations, chemoembolization, therapeutic, SN, 5-FU

\section{Introduction}

Liver cancer, ${ }^{1,2}$ including hepatocellular carcinoma (HCC), cholangiocarcinoma, and other hepatic malignancies, is the sixth most common malignant tumor. Among primary liver cancers, HCC is one of the most common, according to cancer statistics updated in 2019, the estimated number of new HCC case ranks at 14th, while the estimated number of deaths from HCC ranked 5th among cancer-related 
deaths. Currently, hepatic resection is the main treatment for patients with early-stage $\mathrm{HCC}$, while transarterial chemoembolization (TACE) is preferred for patients with middle- and advanced-stage tumors; ${ }^{3}$ however, most traditional hepatic artery embolization agents have disadvantages, including low embolic selectivity, incomplete embolization, and insufficient embolization time, among others. ${ }^{4,5}$ Phytantriol (PYT) ${ }^{6,7}$ is an amphiphilic molecule with both hydrophilic hydroxyl groups and hydrophobic carbon chains. When PYT is dispersed in a small amount of water, a very low viscosity magnetic isotropic solution is formed, referred to as a precursor solution, which has the advantages of good mobility and easy injection; however, when PYT is dispersed in sufficient water and other organic solvents, it can form a high viscosity, rigid liquid crystal gel, which is non-toxic and biocompatible. Hence, the PYT precursor solution has the potential for use in TACE, and has been the subject of several reports. ${ }^{8-11}$

As the most commonly used uracil anti-metabolite drug, 5-Fluorouracil (5-FU) ${ }^{12-14}$ has a broad spectrum of anti-tumor effects. Further, 5-FU is the most widely used drug for treatment of HCC, and has clear therapeutic effects, making it the first choice for treatment of advanced HCC; however, minimal bioavailability, low selectivity, obvious side effects, and drug resistance limit its clinical application. Thus, there is an urgent need to explore alternative therapeutic approaches to improve patient outcomes. Sinomenine $(\mathrm{SN})^{15}$ is the main active alkaloid extracted from Sinomenium acutum; it has anti-inflammatory, analgesic, immunosuppressive, antihypertensive, and antiarrhythmic effects, and is primarily used for treatment of rheumatoid arthritis, chronic gastritis, morphine addiction, and other diseases. Recently, SN has been demonstrated to have strong and extensive anti-tumor effects, ${ }^{16,17}$ and when used in combination with 5-FU, achieves superior efficacy. Further, some studies have demonstrated that $\mathrm{SN}$ has potential as a 5-FU-based chemotherapy sensitizer; ${ }^{18-20}$ however, the effects of SN combined with 5-FU on liver cancer cells have not previously been reported.

Our previous studies have shown that liquid crystal gels based on PYT can be used in the articular cavity, ${ }^{21}$ skin, ${ }^{22}$ and periodontitis; ${ }^{23}$ however, no systematic evaluation of the biocompatibility of PYT has been conducted. The primary objective of the present study was to construct a liquid embolic agent, based on PYT, using SN and 5-FU as model drugs, and investigate its biocompatibility, anticancer activity, and drug release, to assess its feasibility for use in TACE as a new therapeutic modality for patients with liver cancer.

\section{Materials and Methods}

\section{Materials}

Ethanol was provided by Sinopharm Chemical Reagent Co., Ltd. (Hefei, China). PYT (GC > 95\%) was purchased from Tokyo Chemical Industry Co., Ltd. (Tokyo, Japan). SN was supplied by Chengdu Must Biotechnology Co., Ltd. (Chengdu, China). 5-FU was produced by Shanghai Ika Biotechnology Co., Ltd. (Shanghai, China). Sterilized water for injection was purchased from Shanghai Xinyi Jinzhu Pharmaceutical Co., Ltd. (Shanghai, China). Methanol (chromatographic grade) was purchased from Sigma-Aldrich (Shanghai, China). Urethane (purity > 99.6\%) was purchased from Shanghai TargetMol Biotechnology Co., Ltd. (Boston, USA). The HepG2 cell line was purchased from JENNIE-O Biological Technology Co., Ltd. (Guangzhou, China).

All animals were purchased from the Experimental Animal Center of Anhui Medical University (Hefei, China), and all procedures were approved by the Committee on Ethics of Animal Experiments of Anhui University of Chinese Medicine, and conducted in compliance with the National Institutes of Health Guide for the Care and Use of Laboratory Animals.

\section{Preparation of Precursor Formulations}

According to the literature ${ }^{8-11}$ and previous research, ${ }^{21}$ three solutions with ratios of PYT:ethanol:water $(\mathrm{w} / \mathrm{w})$ of 64:16:20 $\left(\mathrm{P}_{1}\right)$, 48:32:20 $\left(\mathrm{P}_{2}\right)$, and 16:64:20 $\left(\mathrm{P}_{3}\right)$, were selected for use in development of a TACE carrier. Briefly, PYT and ethanol were mixed in a water bath at $37^{\circ} \mathrm{C} \pm 0.5^{\circ} \mathrm{C}$, and the designated amounts of water simultaneously preheated. Melted PYT and ethanol were vortexed, to generate a uniform oily phase, which was then vortex-mixed with the prescribed amount of water. Mixtures were then centrifuged at $3000 \mathrm{rpm}$ for $10 \mathrm{~min}$, to obtain the precursor liquids. Drug-loaded preparations (drug-loaded P1, drug-loaded P2, and drug-loaded P3) were formulated by first dissolving the drugs in water, and then performing the operations described above. All samples were equilibrated at room temperature for at least one week and filtered through a sterile filter $(0.22 \mu \mathrm{m})$, prior to use in experiments.

\section{Hemolysis Test}

Preparation of Red Blood Cell Suspensions

In vitro hemolysis assays were conducted using red blood cells (RBCs). The hemolytic activity of samples was 
analyzed by quantification of the released hemoglobin by spectrophotometric measurement. Briefly, one New Zealand rabbit (male, $2.2 \mathrm{~kg}$ ) was used in this study. We collected $18 \mathrm{~mL}$ blood by heart puncture, and placed it into a preprepared centrifuge tube, containing $2 \mathrm{~mL} \mathrm{3.8 \%} \mathrm{sodium}$ citrate, to prepare fresh anticoagulated blood. Blood was slowly stirred using a glass rod to remove fibrin, then diluted in physiological saline (PS) at a 1:1 volume ratio, and centrifuged at $3000 \mathrm{rpm}$ for $10 \mathrm{~min}$. The supernatant was removed, the remaining sample diluted 1:10 in PS, and then purified and washed by centrifugation (3000 rpm, $10 \mathrm{~min}, 5$ times) to collect the RBCs. Finally, precipitated RBCs were diluted with PS to prepare a $2 \%$ RBC suspension. ${ }^{24}$

\section{Experimental Design}

Before the experiment, fluid extracted from the precursor solution $^{25}$ was prepared by adding approximately $0.5 \mathrm{~g}$ of drug-free precursor solution in $5 \mathrm{~mL}$ PS, incubating at $37^{\circ}$ $\mathrm{C}$ for $72 \mathrm{~h}$, then filtering through a sterile filter $(0.22 \mu \mathrm{m})$. To control for the effects of other components in the precursor solution, PYT, 64\% ethanol, 32\% ethanol, and $16 \%$ ethanol were treated in the same manner as described above. Groups used for the experimental design of the hemolysis experiment are shown in Table 1. Briefly, $1.5 \mathrm{~mL}$ of extracts were added to tubes labeled $\mathrm{A}-\mathrm{G}$, with $1.5 \mathrm{~mL}$ PS in test tube $\mathrm{H}$, as a negative control, and $1.5 \mathrm{~mL}$ distilled water in test tube I, as the positive control group. Then, $2 \%$ RBCs suspension was added into each tube, the mixtures gently vortexed, and incubated for $4 \mathrm{~h}$ at $37^{\circ} \mathrm{C}$. All samples were analyzed in triplicate.

Table I Hemolysis Experiment Group Design

\begin{tabular}{|l|l|l|l|l|l|l|l|l|l|}
\hline \multirow{2}{*}{$\begin{array}{l}\text { Extraction } \\
(\mathbf{m L})\end{array}$} & \multicolumn{7}{|c|}{ Test Tube Number } \\
\cline { 2 - 10 } & A & B & C & D & E & F & G & H & I \\
\hline PI & 1.5 & 0 & 0 & 0 & 0 & 0 & 0 & 0 & 0 \\
P2 & 0 & 1.5 & 0 & 0 & 0 & 0 & 0 & 0 & 0 \\
P3 & 0 & 0 & 1.5 & 0 & 0 & 0 & 0 & 0 & 0 \\
PYT & 0 & 0 & 0 & 1.5 & 0 & 0 & 0 & 0 & 0 \\
I6\% Ethanol & 0 & 0 & 0 & 0 & 1.5 & 0 & 0 & 0 & 0 \\
$32 \%$ Ethanol & 0 & 0 & 0 & 0 & 0 & 1.5 & 0 & 0 & 0 \\
$64 \%$ Ethanol & 0 & 0 & 0 & 0 & 0 & 0 & 1.5 & 0 & 0 \\
PS & 0 & 0 & 0 & 0 & 0 & 0 & 0 & 1.5 & 0 \\
Distilled & 0 & 0 & 0 & 0 & 0 & 0 & 0 & 0 & 1.5 \\
Water & & & & & & & & & \\
2\% RBCs & 1.5 & 1.5 & 1.5 & 1.5 & 1.5 & 1.5 & 1.5 & 1.5 & 1.5 \\
Suspension & & & & & & & & & \\
\hline
\end{tabular}

\section{Determination of Hemolysis Rate}

First, the presence or absence of hemolysis in each group of tubes was observed by visual inspection of RBC fragmentation and sedimentation. If RBCs sank, tubes were gently shaken to determine whether they could be resuspended and assess whether agglutination had occurred. Next, tubes were centrifuged at $1000 \mathrm{rpm}$ for $10 \mathrm{~min}$, $150 \mu \mathrm{L}$ aliquots of supernatant form each sample transferred to a 96-well plate, and absorbance recorded using a microplate reader (Biotek, ELX800, USA). The maximum absorption wavelength $\left(\lambda_{\max }\right)$ was determined by UV/Vis spectroscopy as follows: supernatants from the positive control group were placed in quartz cuvettes with a $1 \mathrm{~cm}$ path in a total volume of $2.0 \mathrm{~mL}$, then $\mathrm{UV} /$ Vis spectra (200-800 $\mathrm{nm}$ ) were recorded to determine the $\lambda_{\max }$ value. To express the in vitro hemolytic properties of our samples, the percentage of hemolysis was calculated as follows: ${ }^{26}$

Hemolysis $(\%)=\left(\mathrm{S}_{\text {absorbance }}-\mathrm{H}_{\text {absorbance }}\right) /\left(\mathrm{I}_{\text {absorbance }}-\right.$ $\mathrm{H}_{\text {absorbance) }} \times 100$

where $\mathrm{S}_{\text {absorbance, }} \mathrm{H}_{\text {absorbance, }}$ and $\mathrm{I}_{\text {absorbance }}$ refer to the absorbance values of the sample, negative control group, and positive control group, which were the mean values of measured absorbance from test tubes $\mathrm{A}-\mathrm{G}, \mathrm{H}$, and $\mathrm{I}$, respectively.

\section{Observation of Red Blood Cell Morphology}

The sediment collected in the tubes by centrifugation (as described above) was resuspended in PS, a drop placed on a glass coverslip, and another coverslip placed over it. The coverslips were then gently pulled apart horizontally and allowed to air dry. This technique provides uniform smear thickness and staining. Smears were then flooded with Wright's stain for $2 \mathrm{~min}$, followed by flooding with water for $4 \mathrm{~min}$, drying, mounting on a glass slide, and examination by light microscopy.

\section{In vivo Injectability and Histocompatibility} Subcutaneous injection in Sprague Dawley rats is routinely used to characterize foreign body reactions to polymeric biomaterials. The size of the animals allows for clinically relevant injection volumes $(500-1000 \mu \mathrm{L})$ to be tested; ${ }^{27}$ hence, the foreign body response to the precursor formulations created using varying concentrations of PYT/ethanol $/ \mathrm{H}_{2} \mathrm{O}$ was assessed, using a subcutaneous pouch model in Sprague-Dawley rats (male, $200 \pm 20 \mathrm{~g}$ ). The precursor formulations were sterilized before injection as liquid embolic agents. Rats were anesthetized by 
intraperitoneal injection of pentobarbital sodium solution (30 mg/kg). There were four experimental groups: P1, P2, and P3, which were injected with $500 \mu \mathrm{L}$ of the indicated solutions, and PS, which was injected with $500 \mu \mathrm{L}$ PS, as a negative control.

The subcutaneous tissue surrounding the hydrogel was evaluated by histochemical staining. In brief, rats were sacrificed at the indicated time points and 0.5 $\mathrm{g}$ subcutaneous tissue samples from close to the hydrogel cut into fragments and fixed in paraformaldehyde solution at $4^{\circ} \mathrm{C}$ for further analysis. Tissue samples were subjected to hematoxylin-eosin (HE) staining to determine the degree of the inflammatory response. All images were acquired using a general optical microscope.

\section{In vitro Cytotoxicity}

The in vitro cytotoxicity of samples was determined by MTT assay. The principle of this assay is based on the fact that succinate dehydrogenase in the mitochondria of living cells can reduce exogenous MTT to water-insoluble bluepurple crystalline formazan, which is deposited in cells, while dead cells cannot perform this function. Dimethyl sulfoxide (DMSO) can dissolve the formazan in the cells, and its light absorption value is then measured using an enzyme-linked immunosorbent assay at a wavelength of $540 \mathrm{~nm}$, with the results indirectly reflecting the number of living cells.

\section{Cell Culture}

The human hepatoma cell line, HepG2, was used for in vitro cytotoxicity experiments. The cell line was grown in Dulbecco's Modified Eagle medium (DMEM), supplemented with $10 \%$ fetal bovine serum, $100 \mu \mathrm{g} / \mathrm{mL}$ penicillin, and $100 \mu \mathrm{g} / \mathrm{mL}$ streptomycin. Cells were incubated in a saturated humidity incubator $\left(37^{\circ} \mathrm{C}, 5 \% \mathrm{CO}_{2}\right)$, washed with phosphate-buffered saline (PBS), and digested using $0.25 \%$ trypsin to form a single cell suspension. Cells in logarithmic growth phase were tested.

\section{Preparation of Sample Extracts}

With reference to the relevant standards (GB/T 16886.5-2017 and ISO 10993-5:2009), $1 \mathrm{~g}$ of the blank preparation, P1, P2, P3, and PYT were injected into $5 \mathrm{~mL}$ of DMEM, and leached at $37^{\circ} \mathrm{C}$ for $48 \mathrm{~h}$. Then, samples were filtered through $0.22 \mu \mathrm{m}$ sterile membranes, and the filtrates used as stock solutions. Next, the stock solutions were diluted in different proportions $(1: 0 ; 1: 1 ; 1: 2 ; 1: 4$;
$1: 8 ; 1: 16 ; 1: 32 ; 1: 64 ; 1: 128, \mathrm{v} / \mathrm{v})$ of DMEM culture medium, as test solutions.

\section{In vitro Cytotoxicity Assay}

Briefly, $100 \mu \mathrm{L}$ of DMEM was added to the peripheral wells of a 96-well tissue culture microtiter plate, and 100 $\mu \mathrm{L}$ of HepG 2 cell suspension $\left(1 \times 10^{4}\right.$ cells $\left./ \mathrm{mL}\right)$ added to the remaining wells. After incubating for $24 \mathrm{~h}$, the medium was removed and $100 \mu \mathrm{L}$ of the different concentrations of stock solutions diluted with fresh medium added to each well, with 100\% DMEM as a negative control $(\mathrm{n}=3)$. After incubation for $72 \mathrm{~h}, 20 \mu \mathrm{L}$ MTT solution $(5 \mathrm{mg} / \mathrm{m} \mathrm{L}$ ) was added to each well and the cells incubated at $37^{\circ} \mathrm{C}$ for an additional $4 \mathrm{~h}$. Subsequently, the MTT solution was removed, replaced with $100 \mu \mathrm{L}$ DMSO, and the mixture shaken at a low speed for $10 \mathrm{~min}$ on a shaker to fully dissolve the crystalline formazan. The optical density (OD) at $540 \mathrm{~nm}$ of each well was measured using a microplate reader. To express the in vitro hemolytic properties of our samples, cell viability was calculated using the following formula:

Cell viability $(\%)=\mathrm{OD}_{570 \mathrm{~S}} / \mathrm{OD}_{570 \mathrm{~N}} \times 100$

where $\mathrm{OD}_{570 \mathrm{~S}}$ represents the average optical density of each tested sample group, and the $\mathrm{OD}_{570 \mathrm{~N}}$ represents the average optical density of the negative control group. According to the ISO standard (GB/T 16886.5-2017/ISO 10993-5:2009), cell viability was then classified according to percentage of viable cells, to reflect the degree of toxicity of the material as follows: $0,100 \% ; 1,80-$ $100 \%$; 2, 50-80\%; 3, 30-50\%; 4, 0-30\%.

\section{In vitro Anticancer Activity}

To test the in vitro anticancer effects of $\mathrm{SN}$ and/or 5-FU solutions, according to the literature ${ }^{12-20}$ and preliminary experimental results, the stock solution concentration of 5 -FU was $640 \mu \mathrm{g} / \mathrm{mL}$, while that of $\mathrm{SN}$ was $1280 \mu \mathrm{g} / \mathrm{mL}$, and the stock combination solution containing was 640 $\mu \mathrm{g} / \mathrm{mL} 5-\mathrm{FU}+1280 \mu \mathrm{g} / \mathrm{mL}+\mathrm{SN}$. Four groups were included in the experiment as follows: negative control group (only DMEM culture medium added), 5-FU monotherapy group, SN monotherapy group, and the combination group. All solutions were formulated and diluted with DMEM medium at ratios of 1:0, 1:1, 1:2, $1: 4,1: 8,1: 16,1: 32,1: 64$, and $1: 128(\mathrm{v} / \mathrm{v})$, then filtered through a $0.22 \mu \mathrm{m}$ microporous membrane before use in experiments. Cell proliferation was determined in vitro by MTT assay, as described above, except that cell viability was detected at 24,48 , and $72 \mathrm{~h}$. Based on 
the results of these experiments, drug loading of $\mathrm{SN}$ in precursor injections was set to $0.5 \%$, that of $5-\mathrm{FU}$ to $0.25 \%$, and the combined drug loading to $0.5 \%+0.25 \%$ $(\mathrm{SN}+5-\mathrm{FU})$. Drug-loaded precursor solutions were extracted as described above and diluted with the culture solution to obtain a gradient of sample concentrations.

To analyze the drug interaction between $\mathrm{SN}$ and 5-FU, the co-administration efficacy analysis software, CalcuSyn 2.1, was used to analyze the inhibition rates (IRs) according to Chou and Talalay, ${ }^{28,29}$ and combination index (CI) values, a dose-effect curve, and a median-effect plot were obtained. The median-effect plot is a graph of $x=\log (D)$ vs $y=\log (\mathrm{fa} / \mathrm{fu})$, based on the median effect equation (a general equation for the dose-effect relationship):

$\mathrm{fa} / \mathrm{fu}=(\mathrm{D} / \mathrm{Dm}) \mathrm{m}$

where fa is the fraction affected by the dose, fu is the fraction unaffected ( $\mathrm{fu}=1-\mathrm{fa}$ ), D is the drug concentration or dose, Dm is the median-effect dose or concentration (usually expressed as ED50 or IC50), $\mathrm{m}$ is an exponent signifying the sigmoidicity (shape) of the dose-effect curve; $\mathrm{m}=$ $1,>1$, and $<1$ indicate hyperbolic, sigmoidal, and negative sigmoidal shapes, respectively. $\mathrm{CI}$ is a quantitative measure of the degree of drug interaction, in terms of additive effect (CI $=1$ ), synergism $(\mathrm{CI}<1)$, or antagonism $(\mathrm{CI}>1)$, for a given endpoint of the effect measurement.

\section{In vitro Drug Release}

The dialysis membrane method was used to study in vitro drug release. In summary, approximately $0.5 \mathrm{~g}$ of the precursor injection containing $\mathrm{SN}$ and/or 5-FU was injected into a dialysis bag (MWCO 1000), which was suspended in a centrifuge tube containing an appropriate amount of PS, and subjected to a release test in a shaker at constant temperature $\left(37^{\circ} \mathrm{C} \pm 0.5^{\circ} \mathrm{C}, 100 \mathrm{rpm}\right)$. Simultaneously, equal amounts of free $\mathrm{SN}$ and 5-FU solution were treated in the same manner. All of the release medium was removed at predetermined intervals and replenished with an equal amount of fresh isothermal release medium. All samples were filtered through a $0.45 \mu \mathrm{m}$ microporous membrane and assayed using a validated HPLC method ${ }^{22}$ to calculate the cumulative release percentage. Then, the function of the cumulative release percentage and time was drawn to predict the release mechanism.

\section{Statistical Analysis}

All data are expressed as mean $\pm \mathrm{SD}$, and statistical analyses were performed using SPSS 22.0. $\mathrm{P}<0.05$ or $\mathrm{P}<0.01$ were considered to indicate significant differences.

\section{Results}

\section{Precursor Formulations}

As shown in Figure 1, the precursor injections prepared in this study were all colorless, uniform solutions, which transformed into transparent gels after adding excess water at $37^{\circ}$ C, followed by centrifugation at $8000 \mathrm{rpm}$ for $10 \mathrm{~min}$ to remove excess water. The precursor injection and gel were observed under a polarized light microscope, and all showed dark fields, indicating that the precursor injections were isotropic solutions, and that the gels were in cubic phase. ${ }^{21}$

\section{In vitro Hemolysis Test}

Intravascular hemolysis increases the amount of plasmafree hemoglobin, which can cause toxic reactions or affect the kidneys and other organs. Further, the release of phospholipids can promote thrombosis. Moreover, when hemolysis causes a clinically significant decrease in the number of RBCs, it can lead to anemia and impair oxygen-carrying capacity, with secondary effects on the brain and other organs or tissues. ${ }^{30}$ Since precursor injections are used for arterial embolization and direct contact with blood, we performed a red blood cell hemolysis experiment using the precursor injection, to assess its hemocompatibility.

As shown in Figure 2A, the negative control solution (tube I) was red, and there was no $\mathrm{RBC}$ residue at the bottom of the tube; however, the upper solutions of the other groups were colorless and clear, accompanied by sinking RBCs, that could be resuspended by shaking the tube, indicating good hemocompatibility and no RBCs agglutination. RBCs collected in the base of the tube were stained using Wright's dye. RBC fragments, including red cytoplasm and blue nuclei, were observed under the microscope in the negative control group, while the morphology of RBCs in the other groups was normal (Figure 2B). The hemolysis rate of the precursor formulations at 538 or $575 \mathrm{~nm}$ ( $\lambda_{\max }$ at 538 or $\left.575 \mathrm{~nm}\right)$ was lower than 2\% (Figure 2C), while that in the PYT group was much lower $(0.10 \%$ at $538 \mathrm{~nm}$ and $1.15 \%$ at $575 \mathrm{~nm})$, and the hemolysis rate of the different concentrations of ethanol was slightly higher $(<10 \%)$ (Figure $2 \mathrm{D})$.

\section{In vivo Injectability and Histocompatibility}

Protuberances were formed immediately after injection of $0.5 \mathrm{~mL}$ of the precursor solutions into the dorsal skin of rats; however, only P1 generated a circular bulge, while P2 and P3 were dispersed into flakes (Figure 3A). After 15 min, rat skin samples with liquid crystalline gel attached were excised to 


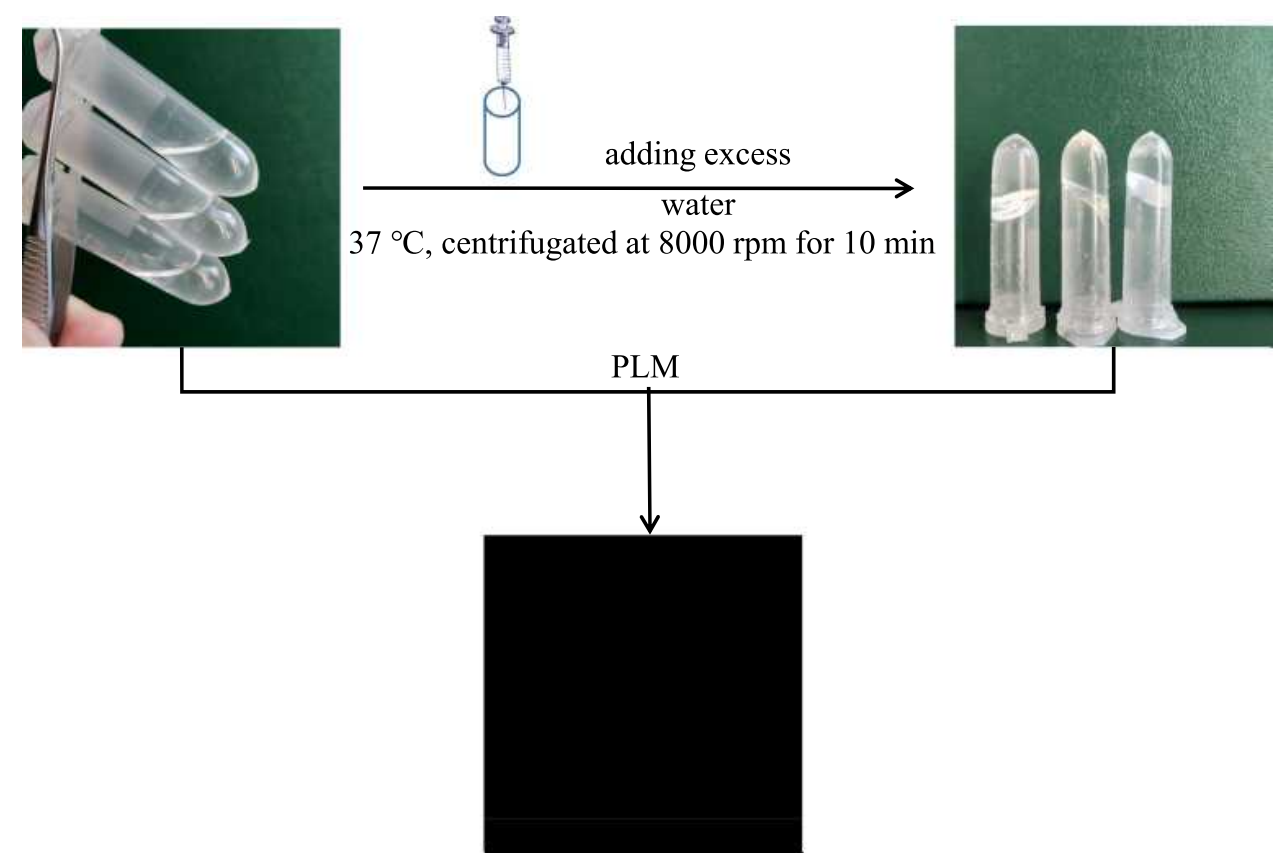

Figure I The appearance of the precursor injection before and after the formation of the in situ liquid crystal gel.

observe the formation of the gel. P1 formed a spherical liquid crystalline gel, while P2 and P3 formed plate-like gels, and P3 spread across a wider area (Figure 3B). Overall, each precursor injection formed a liquid crystal gel, with gelling properties, in vivo. The saline injection also formed a bulge; however, it quickly dispersed (Figure 3A).

Changes of the injection site for each precursor injection were visually observed during subsequent experiments, and pathological changes in tissue samples isolated from the gel injection site analyzed by HE staining (Table 2 and Figure 3C). The results of the histocompatibility experiment revealed erythema and a few inflammatory cells in the P1 group on the first day; however, samples in this group soon returned to normal and no other pathological reactions were observed at later stages, indicating superior histocompatibility. In contrast, the P2 and P3 groups showed strong stimulation from the 1st to 20th days, with skin reactions manifesting as edema, necrosis, and ulcers, and HE staining revealing substantial inflammatory cell infiltration; however, this recovered spontaneously after 20 days, with the P3 group taking longer to recover than the $\mathrm{P} 2$ group.

\section{In vitro Cytotoxicity} In vitro Cytotoxicity of Blank Preparations

To evaluate the cytotoxicity of the precursor injections rapidly and economically, in vitro tests of the precursor injection solutions were conducted using an MTT assay. The results are shown in Figure 4. After $72 \mathrm{~h}$ of incubation, the viability of cells treated with the three types of blank preparation was $>80 \%$ (grade 1 ), and increased with rising dilution ratio, suggesting good cell compatibility of the blank preparation, with only mild cytotoxicity. Furthermore, the cytotoxicity of the PYT material was also measured and the same conclusion reached, supporting the safety of the matrix agents (cell viability $>80 \%$; grade 1).

\section{In vitro Anticancer Activity}

The viability of HepG2 cells after treatment with $\mathrm{SN}$ and/ or 5-FU solutions at different concentrations and times is shown in Figure 5A. As expected, SN and/or 5-FU inhibited cell viability, which decreased with increasing concentrations of $\mathrm{SN}, 5-\mathrm{Fu}$, or the two combined. Incubation time also significantly influenced cell viability, with prolonged incubation time associated with decreased viability. Further, cell viability was more strongly reduced by the combination of SN and 5-FU than either alone, indicating that their inhibitory effects on HepG2 cells may be synergistic. Cell viability rates of extracts treated with different drug-loaded solutions, for various incubation times, at several dilution ratios following application of precursor solutions are presented in Figure 5 (Figure 5B-D). Extracts from each group of drug-loaded preparations 

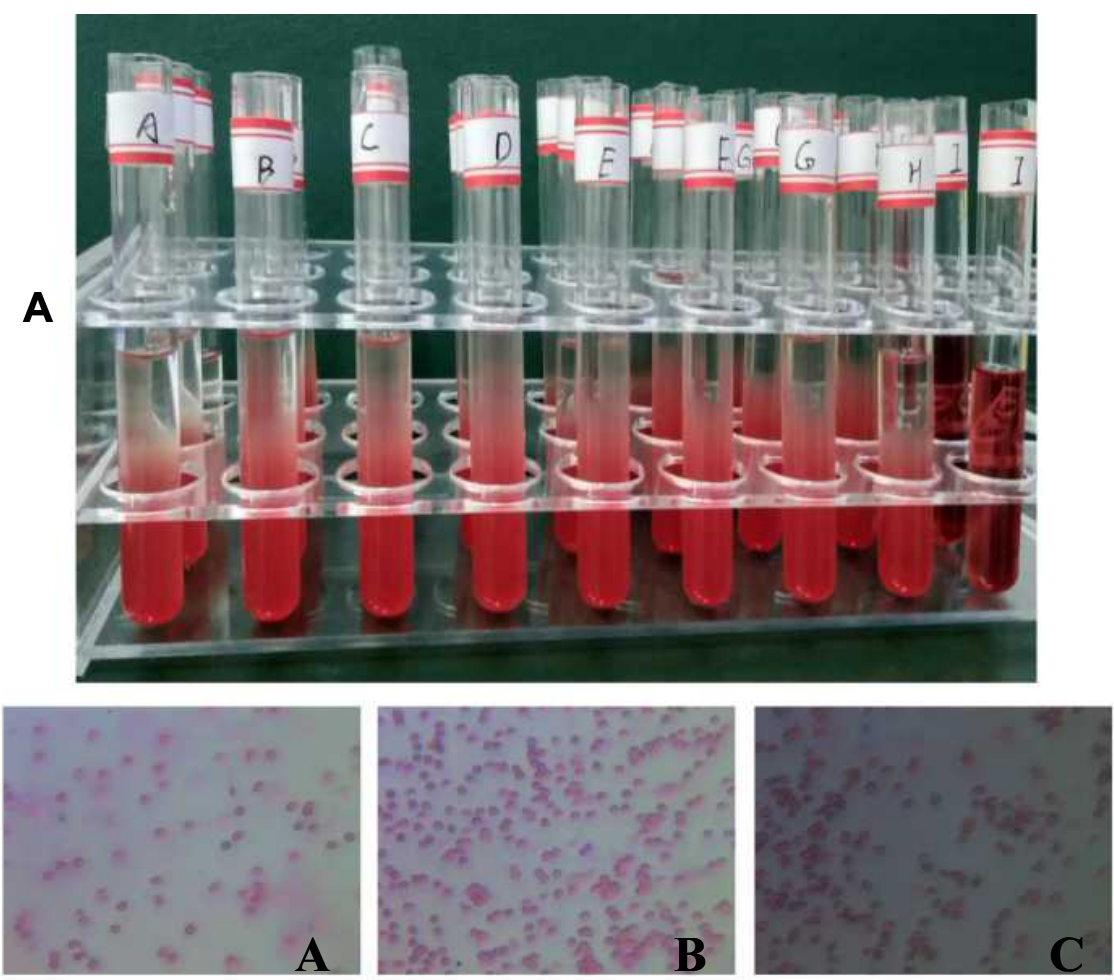

B
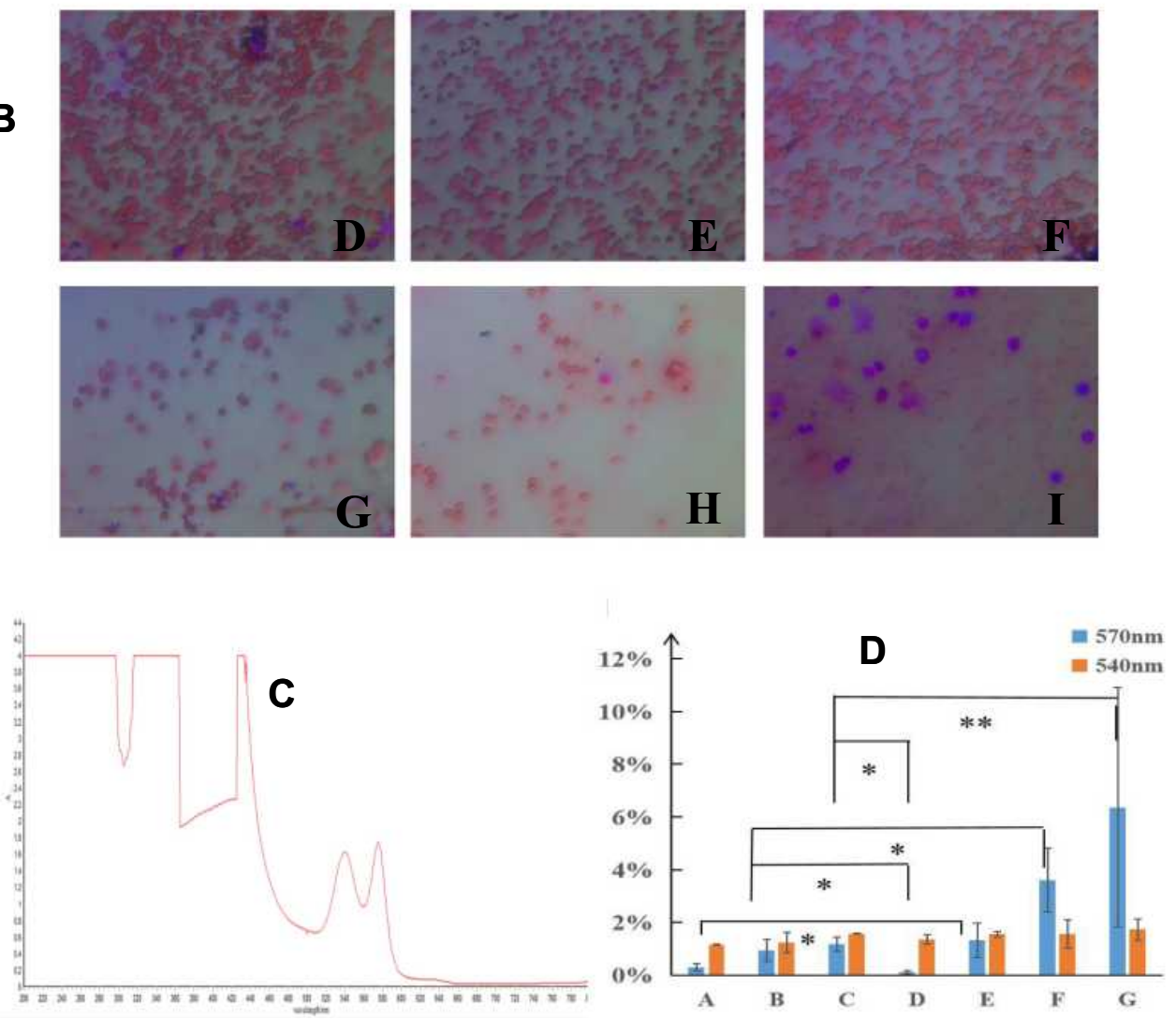

Figure 2 Hemolysis testing: (A) appearance of each group of samples in RCBs solution; (B) RCBs morphology in each group; (C) determination of the $\lambda_{\max }$; (D) hemolysis rate (means $\left.\pm \mathrm{SD}, \mathrm{n}=3 ;{ }^{*}<0.05,{ }^{*} \mathrm{p}<0.01\right)$. 
A

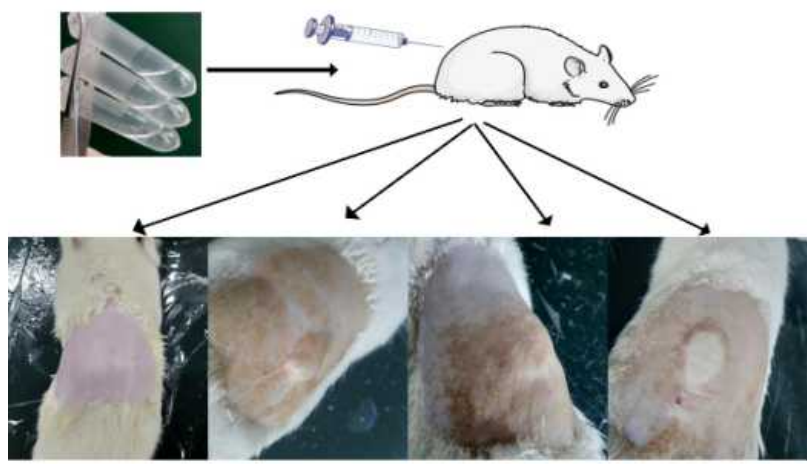

5 min

B

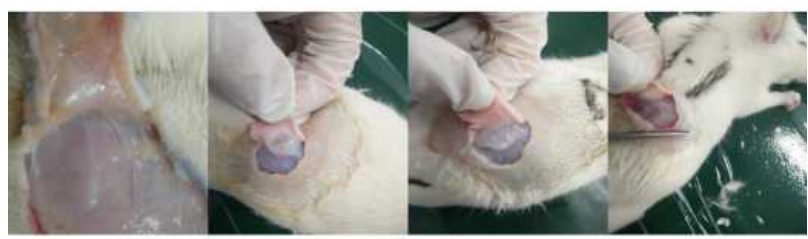

$10 \mathrm{~min}$

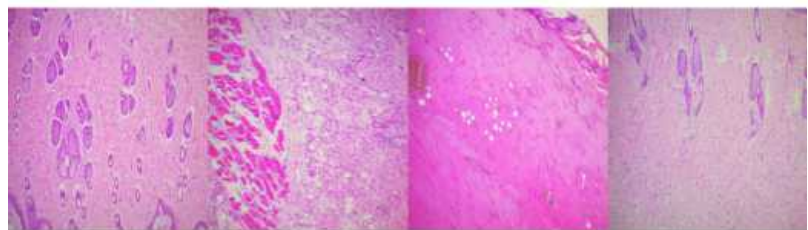

1 day

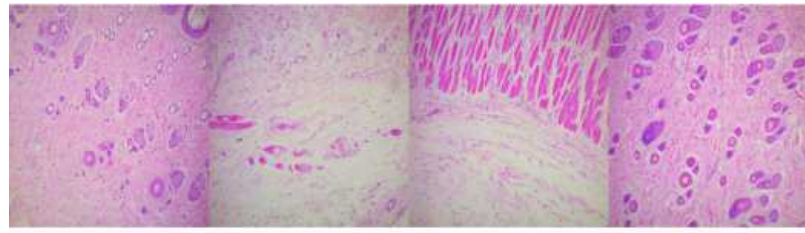

10 day

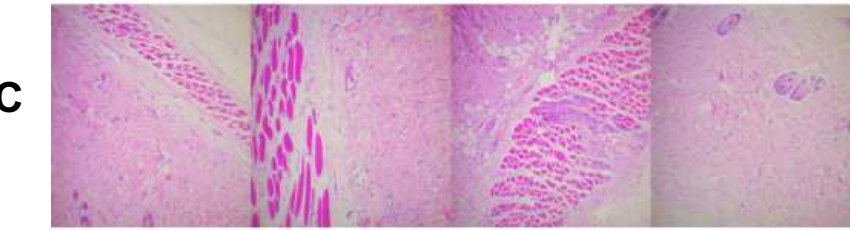

20 day

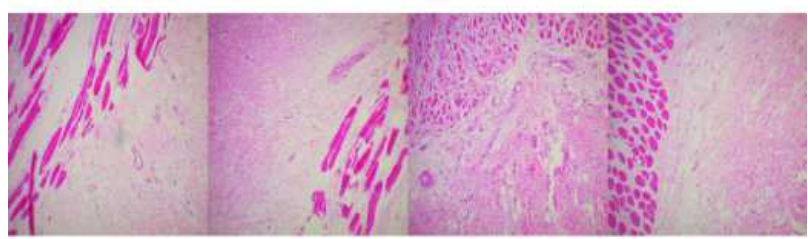

30 day

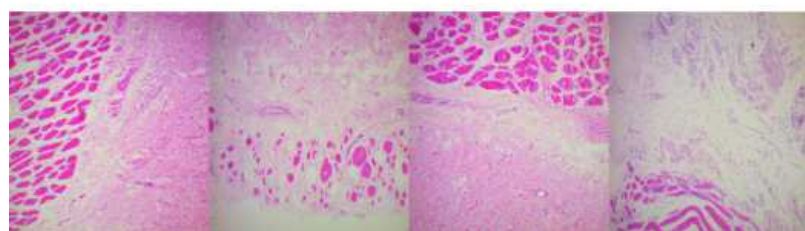

40 day
PC
P1
P2
P3

Figure 3 The results of histocompatibility test: (A) appearance after subcutaneous injection for $5 \mathrm{~min}$; (B) liquid crystal gel state after subcutaneous injection for 15 min; (C) HE staining results of tissue surrounding the liquid crystal gels after subcutaneous injection of the precursor injection into SD rats at the indicated number of days (scale bar: I:100). 
Table 2 The Results of Histocompatibility Experiment

\begin{tabular}{|c|c|c|c|c|c|c|}
\hline \multirow{2}{*}{$\begin{array}{l}\text { Group } \\
\text { Time }\end{array}$} & \multicolumn{2}{|c|}{ PI } & \multicolumn{2}{|c|}{ P2 } & \multicolumn{2}{|c|}{ P3 } \\
\hline & Skin* & HE\# & Skin & HE & Skin & HE \\
\hline I day & ++ & ++ & +++ & ++ & +++ & ++ \\
\hline 10 day & + & + & ++++ & ++++ & ++++ & ++++ \\
\hline 20 day & + & + & ++++ & ++++ & ++++ & ++++ \\
\hline 30 day & + & + & ++ & ++ & +++ & +++ \\
\hline 40 day & + & + & + & + & ++ & ++ \\
\hline
\end{tabular}

Notes: *: + Basically no lesions; ++ erythema, eschar; +++ necrosis, oedema; +++ + corrosion, ulceration. ${ }^{\#}$ : +basically no inflammatory cells; ++A few lymphocytes; +++ lymphocyte infiltration, visible neutrophils; ++++ neutrophil infiltration, visible phagocytic cells.

had clear inhibitory effects on the growth of liver cancer cells. Further, prolonged incubation time and decreased dilution ratio, led to additional reductions in cell viability rates in each group. Furthermore, in vitro cell viability in response to the combined drug-loaded preparation was lower relative to formulations containing each drug alone. This effect was similar to the in vitro anticancer effects of SN and 5-FU solutions; that is, it was concentration-dependent, time-dependent, and synergistic.

Next, we analyzed the interaction between SN and 5-FU using CalcuSyn 2.1 software, generating median-effect plots, dose-effect curves, and algebraic estimates for each drug solution at different times (Figures 6A, $24 \mathrm{~h}$; Figures 6B, $48 \mathrm{~h}$; Figures $6 \mathrm{C}, 72 \mathrm{~h}$ ). The inhibitory effect of each drug solution on HepG2 cells was typically concentration- $(r>$ 0.9 ) and time-dependent. Moreover, the CI values for all incubation times were $<1$ (Table 3). Quantification of CI values at $\mathrm{ED}_{50}(24 \mathrm{~h}, 0.1239 ; 48 \mathrm{~h}, 0.3534 ; 72 \mathrm{~h}, 0.0370)$, $\mathrm{ED}_{75}(24 \mathrm{~h}, 0.0564 ; 48 \mathrm{~h}, 0.2924 ; 72 \mathrm{~h}, 0.4764)$, and $\mathrm{ED}_{90}$ (24 h, 0.0262; 48 h, 0.2794; 72 h, 0.9283) for each drug combination are presented in Table 3. Further, combination of the two drugs led to a significant reduction in Dm value ( $p$ $<0.05 / 0.01)$. Overall, these findings indicate that the inhibitory effects of $\mathrm{SN}$ and 5-FU on $\mathrm{HepG} 2$ cells were synergistic.

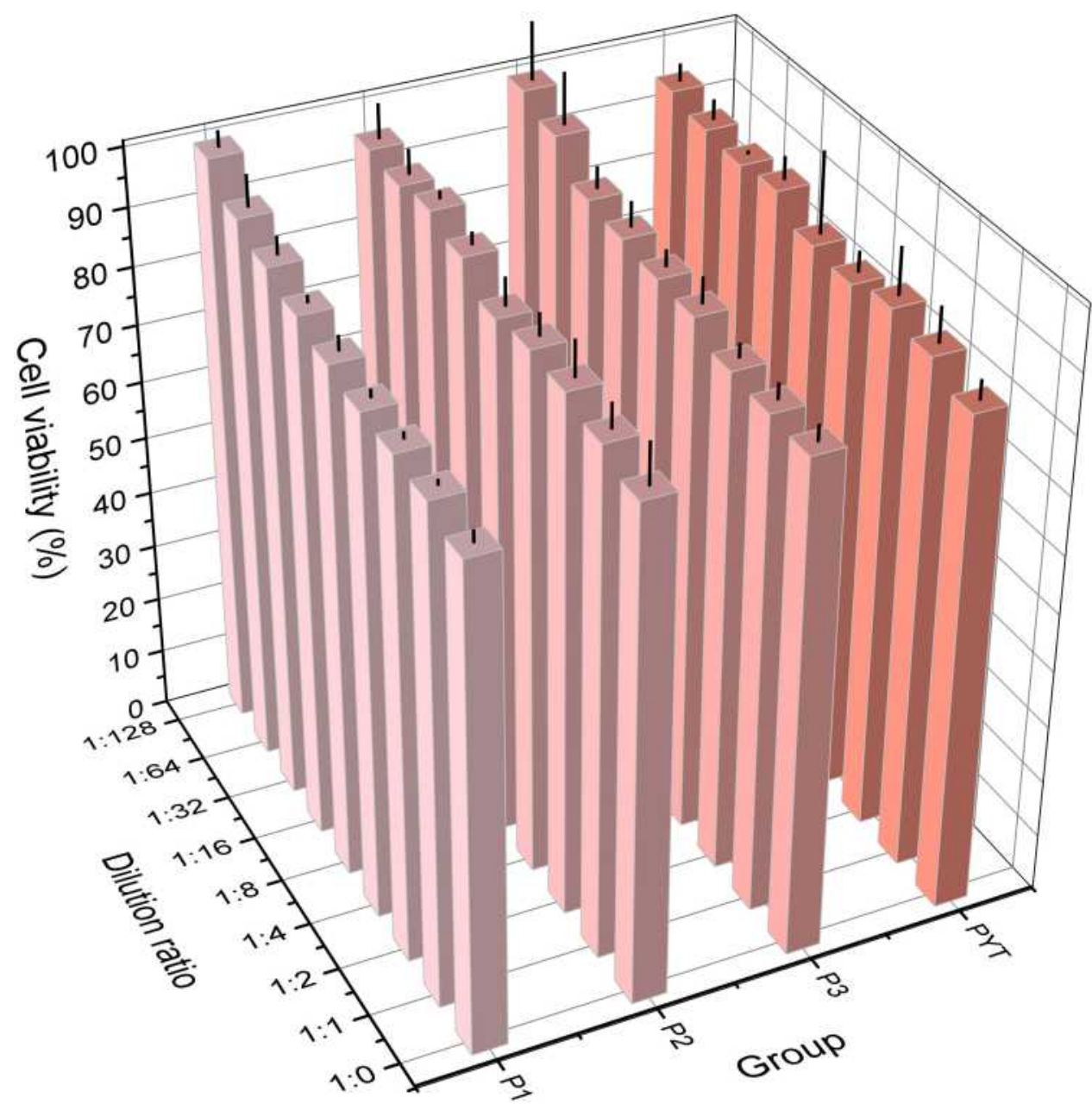

Figure 4 Cytotoxicity of the blank precursor injections and PYT (means $\pm S D, n=3$ ). 


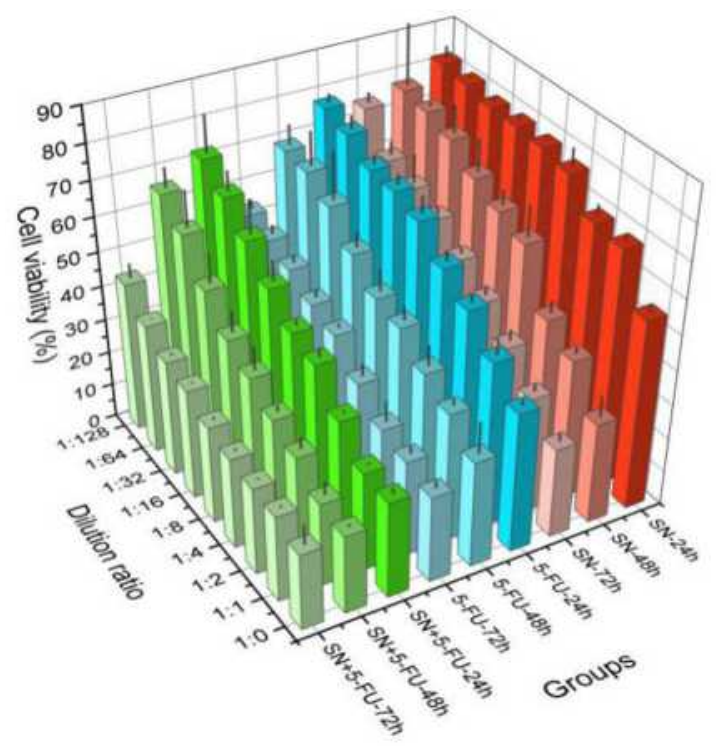

A

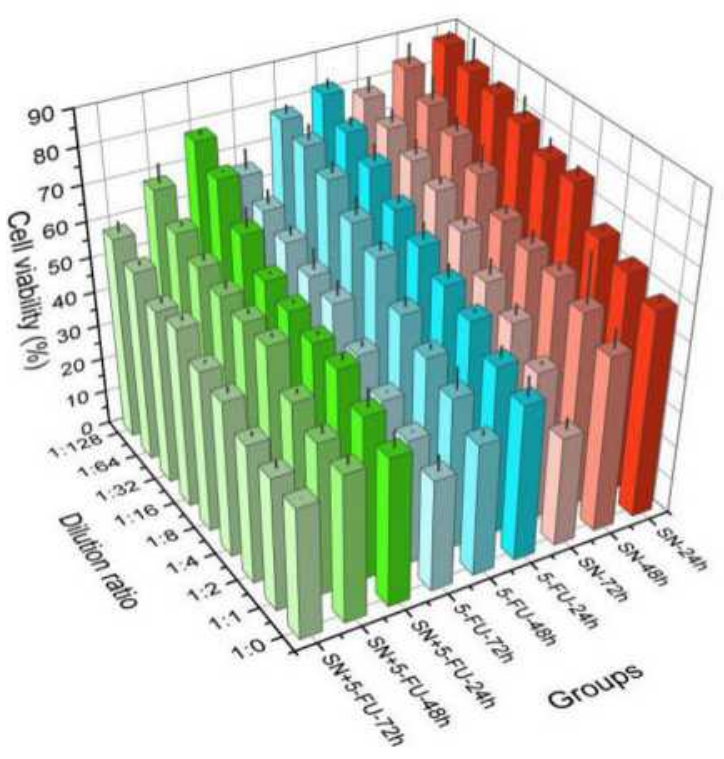

C

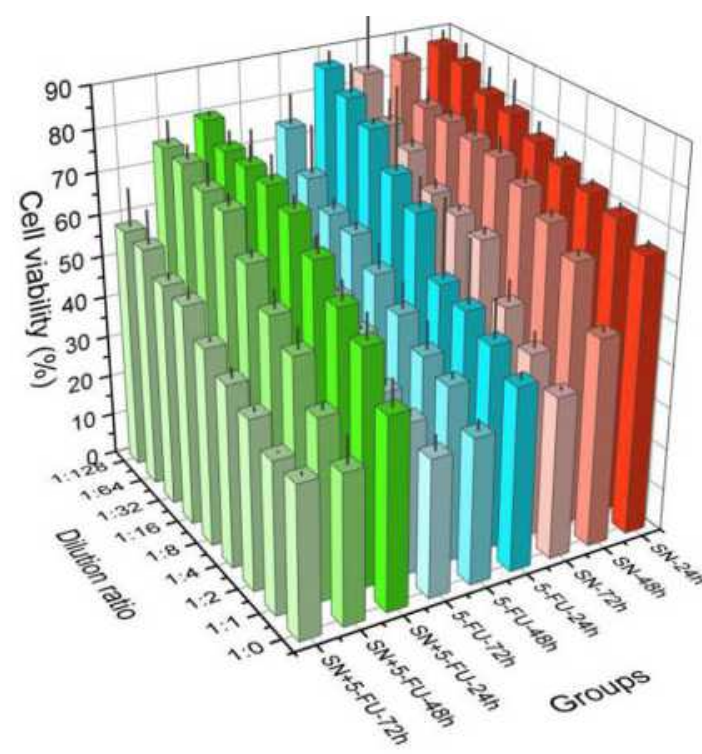

B

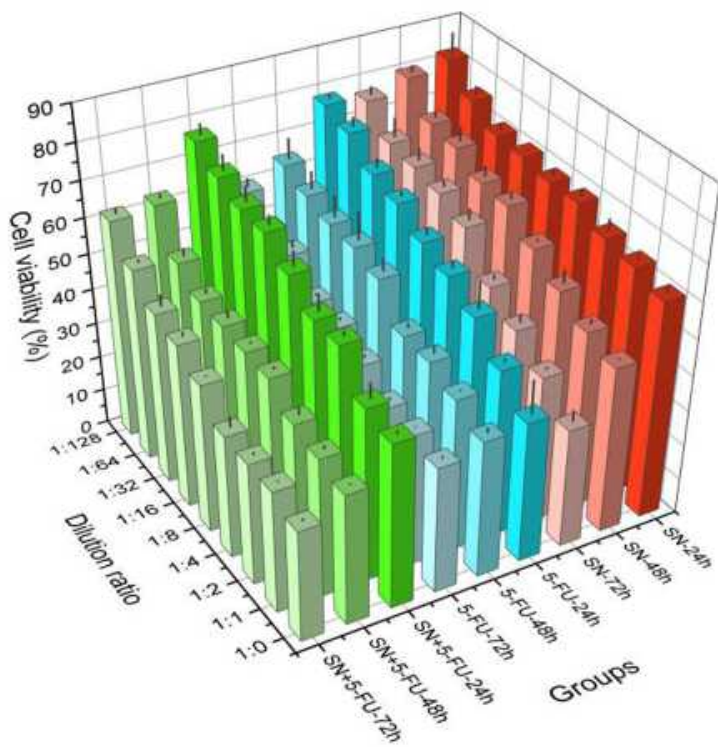

D

Figure 5 The results of in vitro anticancer activity at different time and dilution ratios: (A) SN and/or 5-FU solution; (B) drug-loaded PI; (C) drug-loaded P2; (D) drugloaded P3 (means $\pm \mathrm{SD}, \mathrm{n}=3$; at the same incubation time of the same prescription: $\mathrm{SN}$ or 5 -FU VS SN+5-FU, ${ }^{*} \mathrm{p}<0.05$, ${ }^{* *} \mathrm{p}<0.0 \mathrm{I}$ ).

\section{In vitro Drug Release}

As described above, extracts of drug-loaded preparations suppressed the growth of liver cancer cells, relative to extracts from the blank control preparation. This could be a consequence of release of the drugs from the preparation into to the liver cancer cells; therefore, we studied the in vitro release of the drug-loaded preparations. Each drug-loaded preparation (both double- and single-loaded) contained $0.5 \% \mathrm{SN}$ and/or $0.25 \% 5$-FU. The in vitro release profile of the two drugs within 48 $\mathrm{h}$ was as follows: release of both drugs reached approximately $90 \%$ within $48 \mathrm{~h}$, and there was a significant burst phenomenon at $0.5 \mathrm{~h}$ (where release of the two drugs was $>30 \%$ ) (Figure 7A, SN; Figures 7B, $5-\mathrm{FU})$. The amounts of the drugs released were close to $100 \%$ within $4 \mathrm{~h}$, indicating that the drug-loaded preparations exhibited significant sustained release ( $p$ $<0.05)$.

In addition, to visually express the release behaviors and patterns of the preparations, release data from the first 12 h were fitted to three commonly used mathematical models: Higuchi, first-order, and zero-order equations. Linear 
A

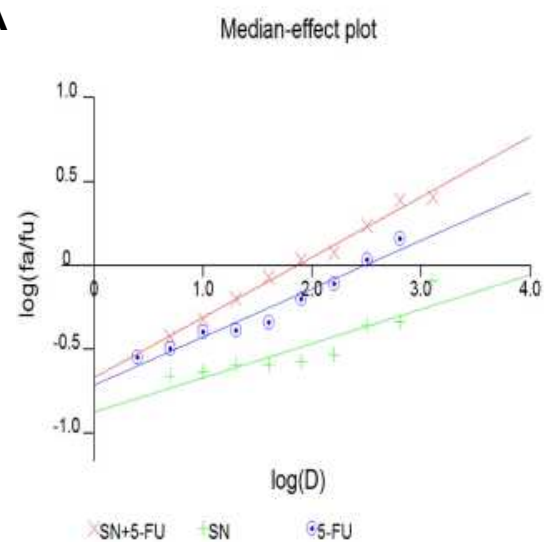

B

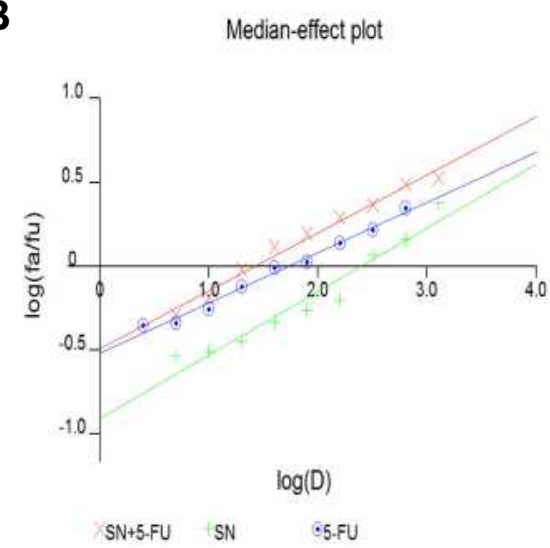

C

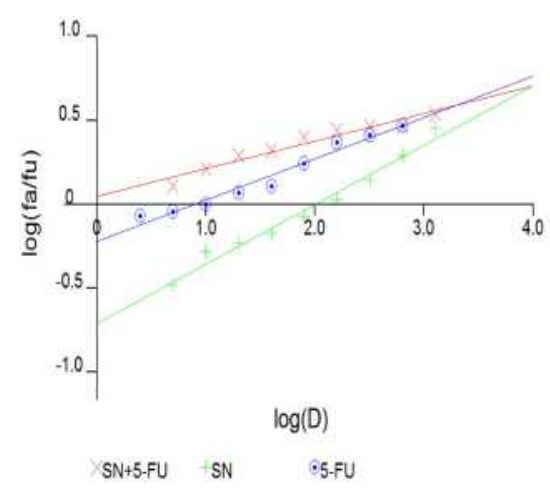

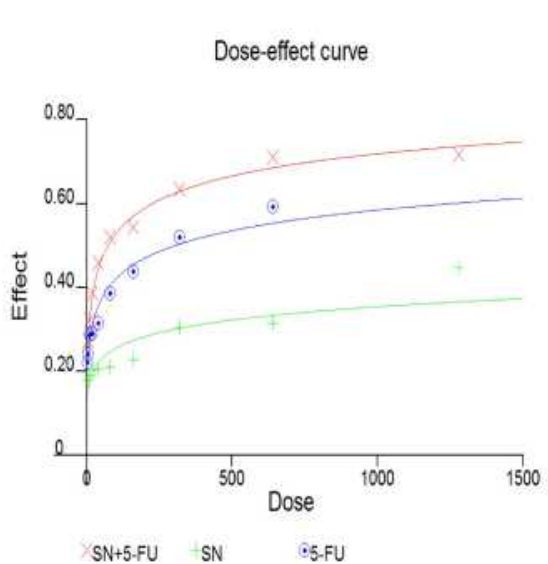

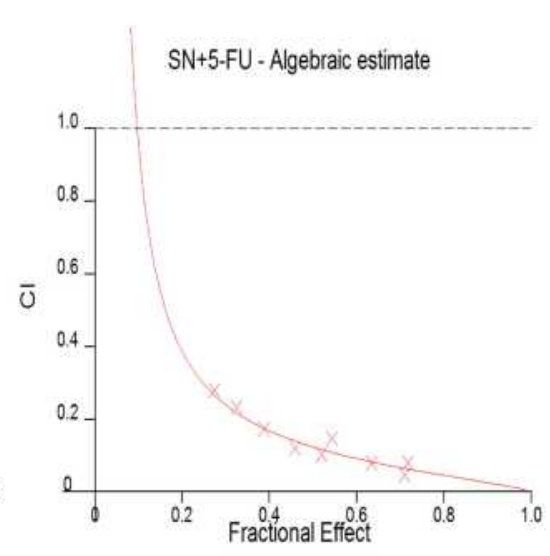

SN+5-FU SN S5-FU
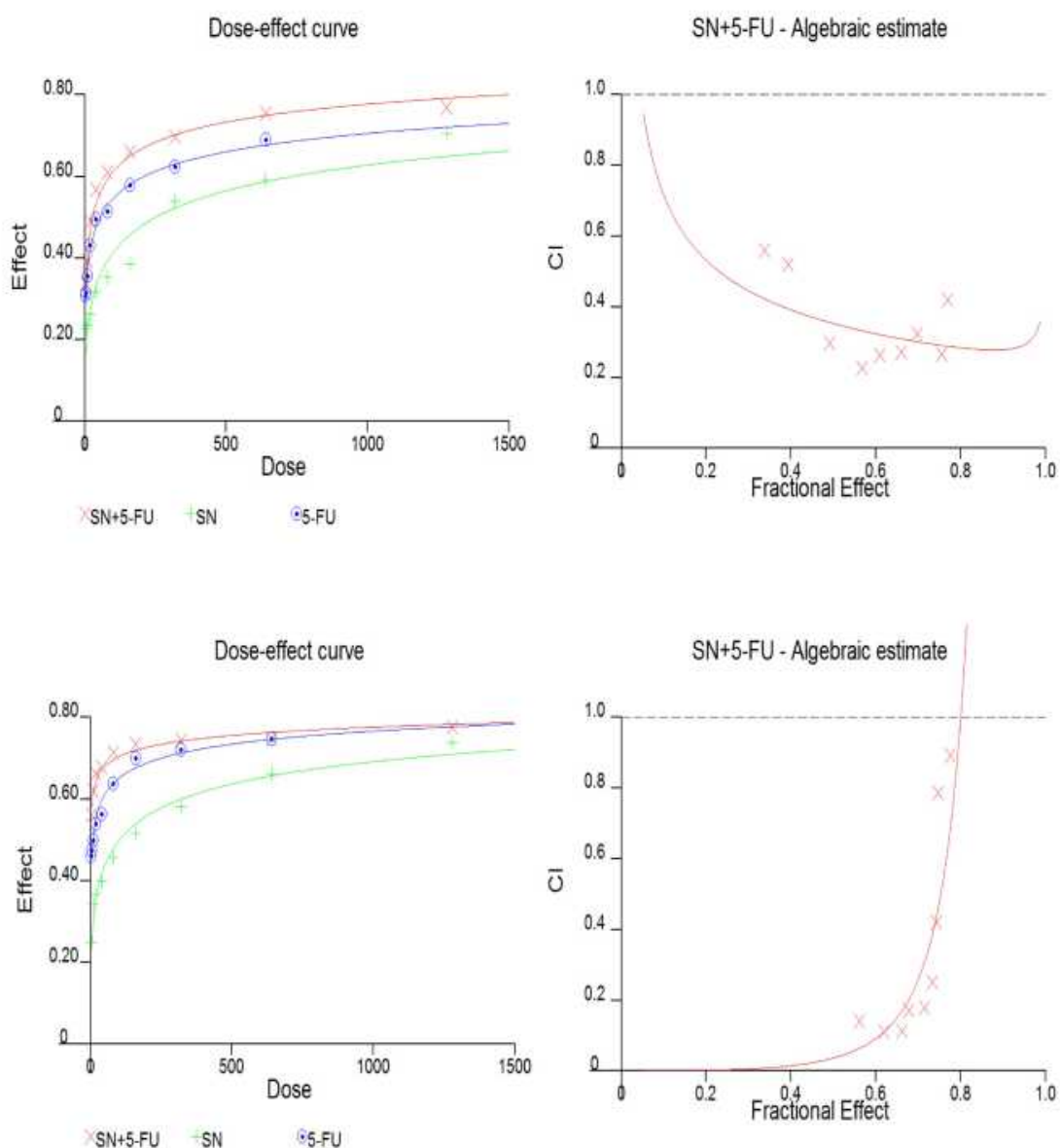

Figure 6 Median-effect plot, dose-effect curve and algebraic estimate generated by CalcuSyn 2.I software for Hep G2 cells treated by various different dilution ratios of SN and/or 5-FU solution at (A) $24 \mathrm{~h},(B) 48 \mathrm{~h}$ and (C) $72 \mathrm{~h}$.

correlation coefficients are presented in Table 4. The $\mathrm{r}$ values for the SN and 5-FU release curves indicated that release of these drugs conforms to first-order kinetics. Although the $r$ value for the Higuchi equation was the largest for an individual group, it did not differ substantially from that for the first-order equation. Overall, the release of the drug was consistent with the first-order equation, indicating that drug release was dominated by swelling. ${ }^{31}$

\section{Discussion}

The precursor injections prepared in this experiment were clear, transparent solutions, that could form cubic liquid 
Table 3 The Results of Hep G2 Cells Treated by Various Different Dilution Ratios of SN and/Or 5-FU Solution by CalcuSyn 2.I Software (Including Cl, r, Dm, M; Compared with the Combined Group of the Same Culture Time, ${ }^{*} p^{<0.05}, *^{*} p<0.01$ )

\begin{tabular}{|c|c|c|c|c|c|c|c|}
\hline \multirow[t]{2}{*}{ Drug } & \multirow[t]{2}{*}{ Time (h) } & \multicolumn{3}{|c|}{ Cl Values at } & \multirow[t]{2}{*}{$\mathbf{r}$} & \multirow[t]{2}{*}{ Dm } & \multirow[t]{2}{*}{$\mathbf{m}$} \\
\hline & & $E D_{50}$ & $E D_{75}$ & $E D_{90}$ & & & \\
\hline \multirow[t]{3}{*}{ SN } & 24 & $\mathrm{~N} / \mathrm{A}$ & $\mathrm{N} / \mathrm{A}$ & $\mathrm{N} / \mathrm{A}$ & 0.9046 & $18908.0000 * *$ & 0.2041 \\
\hline & 48 & $N / A$ & $\mathrm{~N} / \mathrm{A}$ & $N / A$ & 0.9697 & $250.3044 * *$ & 0.3781 \\
\hline & 72 & $N / A$ & N/A & $N / A$ & 0.9990 & $101.8780 * *$ & 0.3538 \\
\hline \multirow[t]{3}{*}{ 5-FU } & 24 & $N / A$ & $N / A$ & $N / A$ & 0.9779 & $303.4755 * *$ & 0.2871 \\
\hline & 48 & $\mathrm{~N} / \mathrm{A}$ & $\mathrm{N} / \mathrm{A}$ & $N / A$ & 0.9927 & $53.7864 *$ & 0.2990 \\
\hline & 72 & $N / A$ & $N / A$ & $N / A$ & 0.9836 & $7.8814^{* *}$ & 0.2456 \\
\hline \multirow[t]{3}{*}{$\mathrm{SN}+5-\mathrm{FU}$} & 24 & 0.1239 & 0.0564 & 0.0262 & 0.9946 & 72.8535 & 0.3585 \\
\hline & 48 & 0.3534 & 0.2924 & 0.2794 & 0.9907 & 26.5875 & 0.3448 \\
\hline & 72 & 0.0370 & 0.4764 & 0.9283 & 0.9725 & 0.5050 & 0.1640 \\
\hline
\end{tabular}

crystals on removal of excess water. Previous rheological experiments $^{32}$ have shown that precursor injections exhibit Newtonian fluid properties at high shear rates, where they exhibit lower viscosity; however, when the cubic liquid

A

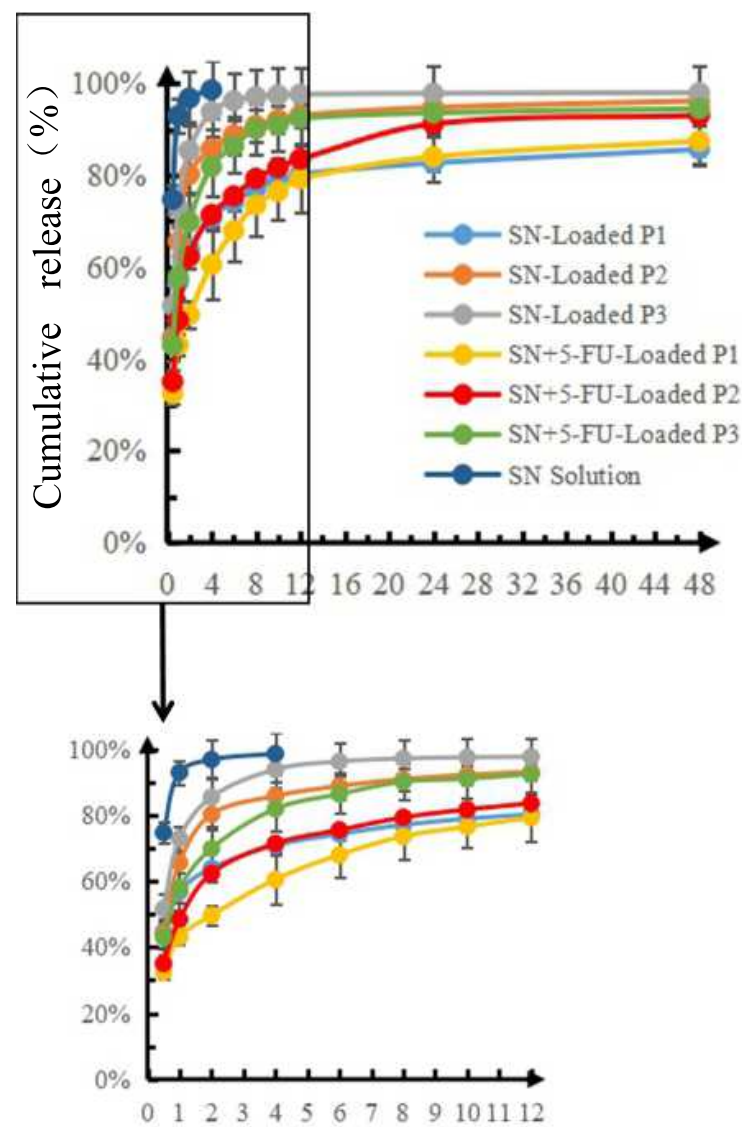

crystals formed, solid and elastic properties were dominant, indicating that all three liquid embolic agents had good injectability and adhesion, as well as being able to resist blood flow clearance.
B
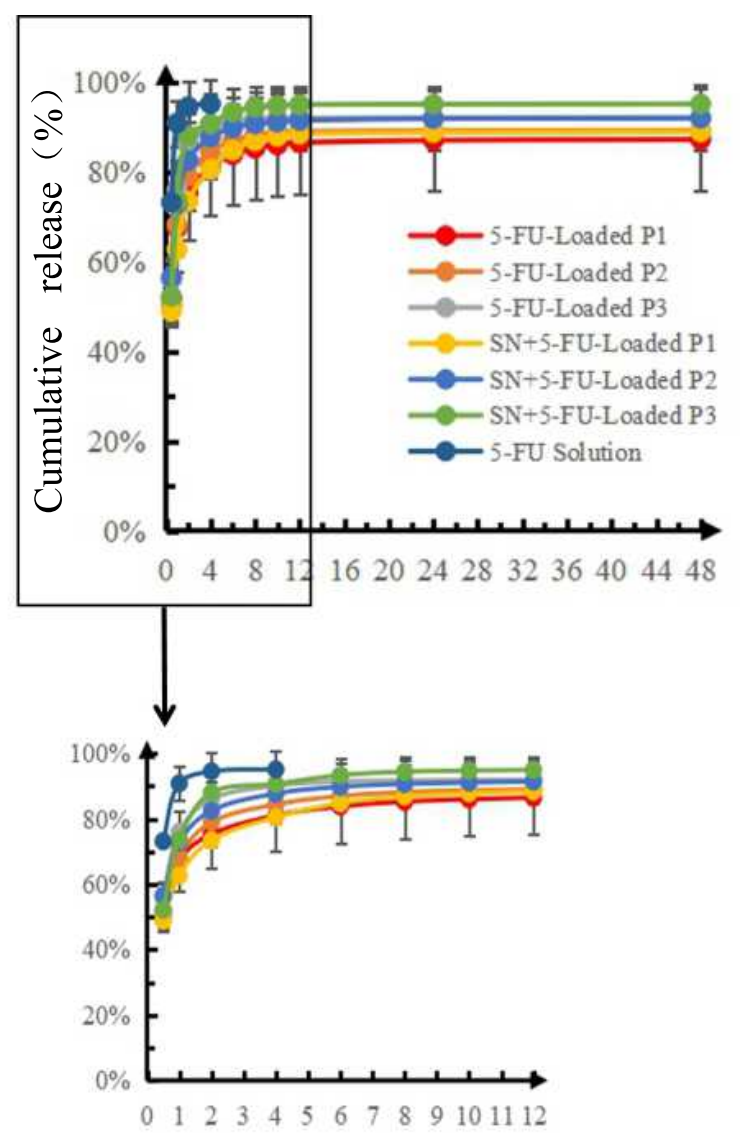

Figure 7 The results of in vitro drug release of SN (A) and 5-FU (B). 
Table $4 r$ Values of Release Curves Fitted by Different Equations

\begin{tabular}{|c|c|c|c|c|}
\hline \multirow[t]{2}{*}{ Drug } & \multirow[t]{2}{*}{ Group } & \multicolumn{3}{|c|}{$\mathbf{r}$} \\
\hline & & Higuchi Equation & First Order Equation & Zero Order Equation \\
\hline \multirow[t]{6}{*}{ SN } & SN-Loaded PI & 0.9507 & 0.9438 & 0.8887 \\
\hline & SN-Loaded P2 & 0.8772 & 0.9228 & 0.7925 \\
\hline & SN-Loaded P3 & 0.8626 & 0.9243 & 0.7704 \\
\hline & SN+5-FU-Loaded PI & 0.9886 & 0.9856 & 0.9498 \\
\hline & SN+5-FU-Loaded P2 & $0.949 \mid$ & 0.9528 & 0.8843 \\
\hline & SN+5-FU-Loaded P3 & 0.9432 & 0.9618 & 0.8736 \\
\hline \multirow[t]{6}{*}{ 5-FU } & 5-FU-Loaded PI & 0.8926 & 0.8917 & 0.8106 \\
\hline & 5-FU-Loaded P2 & 0.8667 & 0.8728 & 0.7776 \\
\hline & 5-FU-Loaded P3 & 0.8078 & 0.8105 & 0.7088 \\
\hline & SN+5-FU-Loaded PI & 0.9241 & 0.9276 & 0.8485 \\
\hline & SN+5-FU-Loaded P2 & 0.8627 & 0.8714 & 0.7723 \\
\hline & SN+5-FU-Loaded P3 & 0.8279 & 0.8736 & 0.7332 \\
\hline
\end{tabular}

To ensure the accuracy of the hemolysis test, we determined the $\lambda_{\max }$ of oxyhemoglobin by full-wavelength scanning. The results showed that the hemolysis rates of the preparations and PYT were well below the safe value (5\%) specified in the relevant standard (GB/T 16886.4-2003/ISO 10993-4:2002), while different concentrations of ethanol resulted in slight hemolysis $(<10 \%)$, indicating that the hemolysis in response to the preparations may be attributable to the ethanol in the solution. Reports indicate that liquid crystal nanoparticles based on PYT exhibit some cytotoxicity and hemolysis, likely because the mesophase structure of the nanostructures is present in biofilms, and when the liquid crystal nanoparticles undergo image transformation, the high concentration of cationic lipids can interact with cell membranes and destroy their integrity, leading to cell lysis and death. ${ }^{33,34}$

In vivo gelation properties and histocompatibility were investigated by subcutaneous injection of precursor solutions into the backs of rats. The results showed that all the precursors could form liquid crystalline gels; however, only P1 formed a spherical shape, while P2 and P3 dispersed into flakes, possibly related to the gelation time of each solution; if the gelation time is faster, gels can be formed in situ before the injection disperses, whereas with slow gelation times, injections exhibit more dispersibility. This suggests that the time of injection should be considered when performing arterial embolization in vivo. If the bolus time is too fast, the injection may flow to other areas before the gel is formed, resulting in ectopic embolization. Histopathological results showed that P1 had good histocompatibility, while P2 and P3 elicited inflammatory responses; however, the latter subsequently recovered spontaneously. High ethanol concentrations generally cause severe inflammation and subsequent necrosis. ${ }^{35,36}$ Comparison of the $\mathrm{P} 1, \mathrm{P} 2$, and $\mathrm{P} 3$ solutions indicates that the inflammation associated with $\mathrm{P} 2$ and $\mathrm{P} 3$ may be caused by excessive ethanol in their solutions. Therefore, P1 is recommended for subsequent in vivo studies.

Unlike traditional methods, the preparations were first diluted with medium before use for in vitro cytotoxicity tests (including anticancer experiments), according to the proposal of Zhai et al. ${ }^{33}$ Injection of precursor solutions is accompanied by release of a large amount of drug during the formation of liquid crystalline gel, ${ }^{8,9}$ as confirmed by our in vitro drug release tests; therefore, if the liquid crystal gel forms before application in an anti-cancer experiment, the amount of drug released may be reduced, potentially influencing the accuracy of the findings. Based on our preliminary results from experiments conducted using direct injections, the water in the medium was absorbed to form a liquid crystal gel, resulting in a decrease in medium content, which may starve the cells and affect their proliferation. To investigate the true state of drug-loaded preparations, we did not induce the precursor injection to form liquid crystal gel before the experiment, rather we directly extracted the injection and then carried out the experiment.

Our results show that SN and 5-FU have clear synergistic effects across a broad concentration range, which were time- and concentration-dependent. Various studies have shown that expression levels of Bax and Bcl-2 are up- and down-regulated, respectively, during the apoptosis 
induced by SIN and 5-FU, ${ }^{18-20}$ indicating that the mechanisms underlying this effect may be associated with increased apoptosis via the mitochondrial pathway and downregulation of thymidylate synthase mRNA expression. It has been suggested that, in the course of 5-FU chemotherapy for patients with liver cancer, addition of SN can greatly reduce the dose of 5-FU required, produce a synergistic effect, and reduce the side effects of 5-FU. Indeed, our data show that use of $\mathrm{SN}$ and 5-FU in our precursor injection for TACE led to good synergistic effects. Further, the blank formulations showed very little cytotoxicity, indicating that the effects of the drug-loaded formulation are likely mainly attributable to drug release. Hence, this combination has potential for treatment of liver cancer.

Since both SN and 5-FU are poorly soluble in water, high drug loading would lead to drug precipitation, particularly when they are used in combination; therefore, we used a drug loading formulation containing $0.5 \% \mathrm{SN}$ and $0.25 \% 5$-FU. The results of the in vitro release test showed that drug release exhibited a burst phenomenon and that higher levels of ethanol in the solution led to increases in the amount and rate of drug release. During the process of water absorption by the precursor solution to form a liquid crystal gel, ethanol in the preparation is exchanged with water, releasing large amounts of the drug into the medium, resulting in the observed burst behavior. In addition, higher proportions of ethanol in solutions are associated with lower viscosity and higher fluidity, which increases the contact area between the preparation and the release medium, leading to elevated drug release. ${ }^{8,33}$ These data suggest that, when this preparation is used in vivo, it can quickly reach the blood concentration required in the human body and produce effective treatment, while simultaneously reducing the side effects of 5-FU.

\section{Conclusion}

The results obtained in this investigation demonstrate that all three of the precursor injections we selected exhibit good biocompatibility and good gelation properties, both in vitro and in vivo. Our data also prove that SN and 5-FU synergistically inhibit the growth of HepG2 cells. Further, we evaluated the in vitro anticancer activity and release of SN- and/or 5-FU-loaded precursor injections and our results show that all three formulations can significantly inhibit the growth of HepG2 cells, while the combined drug-loaded group had synergistic effects. The in vitro release profile was generally consistent with first-order- release kinetics, and demonstrated a significant sustained release effect. The results of this study emphasize the biocompatibility of these three precursor injections for use in TACE and the interaction of $\mathrm{SN}$ and 5-FU in inhibition of HepG2 cell growth, providing a theoretical reference for clinical treatment of liver cancer; however, further research is required, including study of the feasibility of embolization and in vivo pharmacokinetic and pharmacodynamic investigations. We will conduct extensive experimental research in the future, with the aim of developing our liquid embolic agent for use in TACE and improving therapeutic efficacy.

\section{Acknowledgments}

The authors acknowledge financial support from the National Natural Science Foundations of China (81803831, 81873019), the Key University Natural Science Research Project of Anhui province (KJ2018A0301), Anhui Provincial Talents Project for Youth in Universities (No. gxyq2018025), the Anhui Provincial Natural Science Foundation (No. 1408085QH183), and Exploratory Research Projects of Anhui University of Chinese Medicine (RD18200130).

\section{Disclosure}

The authors report no conflicts on interest in this work.

\section{References}

1. Bao J, Yu Y, Chen J, et al. MiR-126 negatively regulates PLK-4 to impact the development of hepatocellular carcinoma via ATR/CHEK1 pathway. Cell Death Disease. 2018;9(10):1045. doi:10.1038/s41419-018-1020-0

2. Dutta R. Recent advances in hepatocellular carcinoma therapy. Pharm Therapeut. 2017;173:106-117. doi:10.1016/j.pharmthera.2017.02.010

3. Nishi M, Saeki I, Yamasaki T, et al. Hemobilia immediately after transcatheter arterial chemoembolization using drug-eluting beads for hepatocellular carcinoma with intrahepatic bile duct invasion. Hepatol Res. 2018;48(4):329-332. doi:10.1111/hepr.12995

4. Zhou X, Li Y, Chen S, et al. Dynamic agent of an injectable and self-healing drug-loaded hydrogel for embolization therapy. Colloid Surface $B \quad$ Biointerfaces. 2018;172:601-607. doi:10.1016/j. colsurfb.2018.09.016

5. Mu L, Chapiro J, Stringam J, Geschwind J-F. Interventional oncology in hepatocellular carcinoma: progress through innovation. Cancer J. 2016;22(6):365-372. doi:10.1097/PPO.0000000000000227

6. Yepuri NR, Clulow AJ, Prentice RN, et al. Deuterated phytantriol A versatile compound for probing material distribution in liquid crystalline lipid phases using neutron scattering. J Colloid Interface Sci. 2019;534:399-407. doi:10.1016/j.jcis.2018.09.022

7. Akbar S, Anwar A, Ayish A, et al. Phytantriol based smart nano-carriers for drug delivery applications. Eur $J$ Pharm Sci. 2017;101:31-42. doi:10.1016/j.ejps.2017.01.035

8. Qin L, Mei L, Shan Z, et al. Phytantriol based liquid crystal provide sustained release of anticancer drug as a novel embolic agent. Drug Develop Industrial Pharm. 2016;42(2):307-316. doi:10.3109/ 03639045.2015.1052079 
9. Qin LZ, Zhang X, Wu LN, et al. Preparation and in vitro embolic efficiency evaluation of hydroxycamptothecine-loaded liquid embolic agent. Acta Pharmaceutica Sinica. 2014;49(7):1069-1075.

10. Han K, Pan X, Chen M, et al. Phytantriol-based inverted type bicontinuous cubic phase for vascular embolization and drug sustained release. Eur J Pharm Sci. 2010;41(5):692-699. doi:10.1016/j.ejps.2010.09.012

11. Han K, Wang Z, Peng X, et al. Transarterial chemoembolization using docetaxel-loaded phytantriol cubic phase precursor for the treatment of hepatocellular carcinoma. J Pharm Sci. 2011;100 (6):2240-2247. doi:10.1002/jps. 22456

12. Liu T, Wu X, Li Y, et al. RBFOX3 regulates the chemosensitivity of cancer cells to 5-fluorouracil via the PI3K/AKT, EMT and cytochrome-C/caspase pathways. Cell Physiol Biochem. 2018;46 (4):1365-1380. doi:10.1159/000489153

13. Cheng M, Ma D, Zhi K, et al. Synthesis of biotin-modified galactosylated chitosan nanoparticles and their characteristics in vitro and in vivo. Cell Physiol Biochem. 2018;50(2):569-584. doi:10.1159/000494169

14. Zhang Y, Jia QA, Kadel D, et al. Targeting mTORC1/2 complexes inhibit tumorigenesis and enhance sensitivity to 5-fluorouracil (5-FU) in hepatocellular carcinoma: a preclinical study of mTORC1/2-targeted therapy in hepatocellular carcinoma (HCC). Med Sci Monitor. 2018;24:2735-2743. doi:10.12659/MSM.907514

15. Chen SP, Sun J, Zhou YQ, et al. Sinomenine attenuates cancer-induced bone pain via suppressing microglial JAK2/STAT3 and neuronal CAMKII/CREB cascades in rat models. Mol Pain. 2018;14:1744806918793232. doi:10.1177/1744806918793232

16. Zhang D, Dong Y, Zhao Y, et al. Sinomenine hydrochloride sensitizes cervical cancer cells to ionizing radiation by impairing DNA damage response. Oncol Rep. 2018;40(5):2889.

17. Fu S, Jin L, Gong T, et al. Effect of sinomenine hydrochloride on radiosensitivity of esophageal squamous cell carcinoma cells. Oncol Rep. 2018;39(4):1601-1608. doi:10.3892/or.2018.6228

18. Zhang JX, Yang ZR, Wu DD, et al. Suppressive effect of sinomenine combined with 5-fluorouracil on colon carcinoma cell growth. Asian Pac J Cancer Prev. 2014;15(16):6737-6743. doi:10.7314/ APJCP.2014.15.16.6737

19. Wang J, Yang ZR, Dong WG, et al. Cooperative inhibitory effect of sinomenine combined with 5-fluorouracil on esophageal carcinoma. World J Gastroenterol. 2013;19(45):8293. doi:10.3748/wjg.v19.i45.8292

20. Liao F, Yang Z, Lu X, et al. Sinomenine sensitizes gastric cancer cells to 5-fluorouracil in vitro and in vivo. Oncol Lett. 2013;6 (6):1604-1610. doi:10.3892/ol.2013.1592

21. Chen Y, Liang X, Ma P, et al. Phytantriol-based in situ liquid crystals with long-term release for intra-articular administration. AAPS Pharm Sci Tech. 2015;16(4):846-854. doi:10.1208/s12249-014-0277-6

22. Chu XQ, Huang J, Li ZG, et al. On the structure and transdermal profile of liquid crystals based on phytantriol. Curr Drug Deliv. 2018;15(10):1439-1448. doi:10.2174/1567201815666180910142516

23. Yang Z, Liang X, Jiang X, et al. Development and evaluation of minocycline hydrochloride-loaded in situ cubic liquid crystal for intra-periodontal pocket administration. Molecules. 2018;23 (9):2275. doi:10.3390/molecules23092275
24. Li HC, Wang DG, Hu JH. Influence of fluoride additions on biological and mechanical properties of $\mathrm{Na}_{2} \mathrm{O}-\mathrm{CaO}-\mathrm{SiO}_{2}-\mathrm{P}_{2} \mathrm{O}_{5}$ glass-ceramics. Mat Sci Eng C-Mat. 2014;35:171-178. doi:10.1016/ j.msec.2013.10.028

25. Han K, Wang ZH, Dong YX, et al. Investigation on biocompatibility and in vivo characteristics of in situ phytantriol liquid crystalline. Chin Pharm J. 2010;45(8):611-616.

26. Gao T, Zeng H, Xu H, et al. Novel self-assembled organic nanoprobe for molecular imaging and treatment of gram-positive bacterial infection. Theranostics. 2018;8(7):1911-1922. doi:10.7150/ thno. 22534

27. Varma DM, Lin HA, Long RG, et al. Thermoresponsive, redox-polymerized cellulosic hydrogels undergo in situ gelation and restore intervertebral disc biomechanics post discectomy. Eur Cell Mat. 2018;35:300-317. doi:10.22203/eCM.v035a21

28. Chou TC. Quantitative analysis of dose-effect relationships: the combined effects of multiple drugs or enzyme inhibitors. $A d v$ Enzym Regul. 1984;22:27-55. doi:10.1016/0065-2571(84)90007-4

29. Ashton JC. Drug combination studies and their synergy quantification using the Chou-Talalay method-letter. Cancer Res. 2015;75 (11):2400. doi:10.1158/0008-5472.CAN-14-3763

30. Giovannini G, Warncke P, Fischer D, et al. Improving colloidal stability of silica nanoparticles when stored in responsive gel: application and toxicity study. Nanotoxicol. 2018;12(5):417. doi:10.1080/ 17435390.2018.1457729

31. Dima C, Pătraşcu L, Cantaragiu A, et al. The kinetics of the swelling process and the release mechanisms of Coriandrum sativum L. essential oil from chitosan/alginate/inulin microcapsules. Food Chem. 2016;195:39-48. doi:10.1016/j.foodchem.2015.05.044

32. Cao JJ, Huang J, Gui SY, et al. [Rheological properties of sinomenine in situ liquid crystal as liquid embolic agent]. China $J$ Chinese Materia Medica. 2018;43(12):2522-2530.

33. Zhai J, Hinton TM, Waddington LJ, et al. Lipid-PEG conjugates sterically stabilize and reduce the toxicity of phytantriol-based lyotropic liquid crystalline nanoparticles. Langmuir. 2015;31 (39):10871-10880. doi:10.1021/acs.langmuir.5b02797

34. Astolfi P, Giorgini E, Gambini V, et al. Lyotropic liquid-crystalline nanosystems as drug delivery agents for 5-fluorouracil: structure and cytotoxicity. Langmuir. 2017;33(43):12369-12378. doi:10.1021/acs. langmuir.7b03173

35. Mason KP, Michna E, Zurakowski D, et al. Serum ethanol levels in children and adults after ethanol embolization or sclerotherapy for vascular anomalies. Radiol. 2000;217(1):127-132. doi:10.1148/radiology.217.1.r00se30127

36. Kazekawa K, Iwata H, Shimozuru T, et al. Nontoxic embolic liquids for treatment of arteriovenous malformations. J Biomed Mat Res. 1997;38(2):79-86. doi:10.1002/(SICI)1097-4636(199722)38:2<79:: AID-JBM1>3.0.CO;2-D
International Journal of Nanomedicine

\section{Publish your work in this journal}

The International Journal of Nanomedicine is an international, peerreviewed journal focusing on the application of nanotechnology in diagnostics, therapeutics, and drug delivery systems throughout the biomedical field. This journal is indexed on PubMed Central, MedLine, CAS, SciSearch ${ }^{\mathbb{R}}$, Current Contents ${ }^{\mathbb{B}} /$ Clinical Medicine,
Journal Citation Reports/Science Edition, EMBase, Scopus and the Elsevier Bibliographic databases. The manuscript management system is completely online and includes a very quick and fair peer-review system, which is all easy to use. Visit http://www.dovepress.com/ testimonials.php to read real quotes from published authors. 